\title{
KMT5A promotes metastasis of clear cell renal cell carcinoma through reducing cadherin-1 expression
}

\author{
ZHEN-ZHONG LIN $^{1 *}$, DE-SONG MING ${ }^{1 *}$, YA-BIN CHEN $^{1}$, JIAN-MING ZHANG ${ }^{1}$, \\ HUI-HUA CHEN ${ }^{1}$, JIAN-JIA JIANG ${ }^{2}$ and ZHI-SHAN ZHANG ${ }^{1}$ \\ Departments of ${ }^{1}$ Clinical Laboratory and ${ }^{2}$ Endocrinology, The First Hospital of Quanzhou \\ Affiliated to Fujian Medical University, Quanzhou, Fujian 362000, P.R. China
}

Received April 14, 2018; Accepted December 17, 2018

DOI: $10.3892 /$ ol.2019.10163

\begin{abstract}
Clear cell renal cell carcinoma (ccRCC) is one of the most common types of kidney cancer and is accompanied by a poor prognosis due to a high potential for metastasis and recurrence. The mechanism of ccRCC metastasis is not well known. N-lysine methyltransferase KMT5A serves a crucial role in the progression of human cancer; however, the function of KMT5A in the development of ccRCCs has not yet been investigated, which has triggered an interest in investigating the potential association between KMT5A and ccRCC. The present study demonstrates for the first time that KMT5A is a driving factor in ccRCC metastasis. The KMT5A expression level was revealed to be significantly higher in ccRCC tissues compared with adjacent normal tissues. Patients with ccRCC whose tumors expressed high levels of KMT5A were demonstrated to have significantly shorter postoperative survival times. In vitro knockdown of KMT5A expression in 786-O cells inhibited cell migration and invasion. KMT5A reduced cadherin-1 (CDH1) protein levels by directly inhibiting its transcription. The CDH1 mRNA levels were inversely correlated with KMT5A expression in ccRCC samples. Patients with high tumor KMT5A or low CDH1 levels had the poorest prognosis with the shortest overall survival (OS) time, and this combination was demonstrated to be an independent prognostic indicator for patient OS time in ccRCC, more accurate than monitoring KMT5A or CDH1 alone. Together, these results indicate that KMT5A serves a vital role in ccRCC development and progression, and it may be a novel target for ccRCC treatment and prevention.
\end{abstract}

Correspondence to: Dr Zhi-Shan Zhang, Department of Clinical Laboratory, The First Hospital of Quanzhou Affiliated to Fujian Medical University, 248 East Street, Quanzhou, Fujian 362000, P.R. China

E-mail: 15859775058@139.com

*Contributed equally

Key words: clear cell renal cell carcinoma, N-lysine methyltransferase KMT5A, metastasis, cadherin-1

\section{Introduction}

Renal cell carcinoma (RCC) arises mainly in the renal parenchyma, and accounts for $>90 \%$ of kidney carcinomas worldwide (1). RCC has the highest mortality rate among the types of genitourinary cancer with cancer statistics for 2018 indicating that RCC constitutes $3 \%$ of adult malignant tumors and accounts for $2 \%$ of all cancer mortalities (2). A significant histological subtype of RCC is clear cell (cc)RCC. Although the treatment method of nephrectomy has improved survival times, advanced ccRCC remains lethal due to tumor metastasis and recurrence (3). Furthermore, the pathogenesis of ccRCC is not well understood and the underlying genetic defects are widespread and complex. The molecular mechanism of ccRCC tumor growth and metastasis is also unclear and its clarification will accelerate the identification of novel targets for treatment and intervention.

N-lysine methyltransferase KMT5A, a member of the SET domain-containing methyltransferase family, specifically catalyzes the monomethylation of histone H4 lysine $20(4,5)$. KMT5A helps maintain genome integrity and regulates cell cycle progression and development (6-11). Previous studies have reported that KMT5A is overexpressed in several tumors, including hepatocellular carcinoma, breast cancer, colorectal cancer, thyroid cancer, esophageal squamous cell carcinoma, melanoma, prostate cancer and ovarian cancer, and promotes tumor progression (12-22). The crucial role of KMT5A in human cancer progression has triggered an interest in investigating the potential association between KMT5A and ccRCC. Whether KMT5A serves a role in ccRCC invasion and metastasis remains unknown.

The present study aimed to investigate the function role of KMT5A in ccRCC development progression. Firstly, the present study analyzed the expression of KMT5A and its clinical significance in the ccRCC tissue samples using transwell assays to evaluate the function of KMT5A in RCC cell migration and invasiveness in vitro. The relationship between KMT5A and CDH1 were detected in the cell line and ccRCC samples, and the association between the combination of KMT5A and CDH1 expression and patient overall survival (OS) time were analyzed. 


\section{Materials and methods}

Tissue samples. Data from 522 ccRCC tissue samples, including gene expression and patient survival information, were obtained from The Cancer Genome Atlas database (https://portal.gdc.cancer.gov/). Additionally, tumor tissue specimens were collected from 20 patients with ccRCC who underwent resection treatment at the First Hospital of Quanzhou Affiliated to Fujian Medical University during 2016. These patients assigned randomly by computer (including 10 females and 10 males, with an age range of 50-70 years). The present study was approved by the ethics committee of the First Hospital of Quanzhou Affiliated to Fujian Medical University.

Plasmid constructs. Lentiviral vector pLKO.1-TRC (Addgene plamid 10879) was used for generating recombinant lentiviruses. In order to achieve RNA interference of KMT5A, DNA fragments encoding hairpin precursors for shKMT5A\#1 (5'-CGCAACAGAATCGCAAACTTA-3') and shKMT5A\#2 (5'-GAATCGCAA ACTTACGGATTT-3') (GENEWIZ, Suzhou, China) were inserted into pLKO.1-TRC. A scrambled siRNA precursor (SCR, 5'-TTCTCCGAACGTGTCACG T-3') (GENEWIZ, Suzhou, China) was used as negative control. For re-expression of KMT5A in the ccRCC and papillary RCC cells with silenced KMT5A expression, the full-length KMT5A cDNA was cloned into pWPI.1 (Addgene plamid 12254). Synonymous mutations were introduced an shKMT5A\#1 (5'-CGCAGCAAA ACCGTAAGCTCA-3', mutations underlined) (GENEWIZ, Suzhou, China) target sequences in KMT5A coding sequences to create the RNAi resistant shKMT5A-RES plasmid.

Cell lines, transfection and lentiviruses. Human ccRCC 786-O cells, papillary RCC ACHN cells and 293 cells were cultured in Dulbecco's modified Eagle medium (DMEM, 10\% fetal bovine serum, Gibco; Thermo Fisher Scientific, Inc.) and maintained at $37^{\circ} \mathrm{C}$ with $5 \% \mathrm{CO}_{2}$ condition. pMD2.G $(2.5 \mu \mathrm{g}$, Addgene plamid 12259) and pSPAX2 (7.5 $\mu \mathrm{g}$, Addgene plamid 12260) used as helper plasmids were together with pLKO.1-based plasmids (10 $\mu \mathrm{g}$, Addgene plamid 10879) to transfected into 293 cells to assemble the recombinant lentiviruses. The transfection efficiency of 293 cells is almost $100 \%$, the transfection efficiency was evaluated using GFP plasmid (Clontech, Mountain View, CA, USA). Transfection was performed using Lipofectamine 2000 Reagent (Themo Fisher Scientific, Inc., Waltham MA, ISA) according to the manufacturers' protocols. At $48 \mathrm{~h}$ later, supernatants from co-transfections were used directly for infection of Human ccRCC 786-O cells.

RNA extraction and RT-qPCR assay. TRIzol (Invitrogen, Thermo Fisher Scientific, Inc.) reagent was used to extract the total RNA from the ccRCC cells (786-O-SCR and 786-O-shKMT5A), and PrimeScript RT reagent kit (TaKaRa Biotechnology Co., Ltd., Dalian, China) was used to convert the mRNA to cDNA at $37^{\circ} \mathrm{C}$ according to the manufacturer's protocol. The corresponding primer sequences were as follows: KMT5A forward, 5'-TCACTCTGTTTCACG CC-3'; and reverse, 5'-CATTCCTCCATCTCATC-3'; CDH1 forward, 5'-GTGAGAGGAATCCAAAGCC-3'; and reverse,
5'-AATGGCAGGGAGTTGGGG-3'; and $\beta$-actin forward, 5'-CCCTGGAGAAGAGCTAC-3'; and reverse, 5'-TAGTTT CGTGGATGCCAC-3'. The qPCR was performed with the following conditions: $95^{\circ} \mathrm{C} / 30 \mathrm{sec}, 40$ cycles of $95^{\circ} \mathrm{C} / 5 \mathrm{sec}$, $60^{\circ} \mathrm{C} / 15 \mathrm{sec}$ and $72^{\circ} \mathrm{C} / 10 \mathrm{sec}$ on MXP3000 cycler (Stratagene, La Jolla, CA, USA) for detecting the relative level of KMT5A expression using SYBR Premix Ex Taq (TaKaRa Biotechnology CO., Ltd.) and quantified by the $2^{-\Delta \Delta C q}$ method using $\beta$-actin as the control and expressed as $2^{\wedge}(-\Delta \Delta \mathrm{Cq})(23)$.

Transwell assays. For transwell invasion assays, matrigel (BD biosciences, Franklin Lakes, NJ, USA) was allowed to polymerize at the base of the top chamber of a 24-well transwell plate ( $8 \mu \mathrm{m}$, Corning Costar Corp, Corning, NY, USA) for $45 \mathrm{~min}$ at $37^{\circ} \mathrm{C} .786-\mathrm{O}$ (SCR, shKMT5A and shKMT5A-RES) and ACHN (SCR, shKMT5A and shKMT5A-RES) cells $\left(1 \times 10^{5}\right.$ cells/well $)$ were starved in serum- and growth factor-free medium for $24 \mathrm{~h}$ and added to the top chambers. The bottom chambers were filled serum-containing medium (10\% fetal bovine serum, Gibco; Thermo Fisher Scientific, Inc.). Cultures were maintained for $48 \mathrm{~h}$, and was followed by the removal of non-invading cells from the upper surface of the membrane with cotton swabs. Cells that had invaded were fixed in $4 \%$ paraformaldehyde and stained with $0.1 \%$ crystal violet for $15 \mathrm{~min}$ at room temperature.

Western blotting. The 786-O (SCR, shKMT5A\#1 and \#2) cells were lysed in RIPA buffer (Thermo Fisher Scientific, Inc.) supplemented with protease inhibitors (Thermo Fisher Scientific, Inc.). The protein concentration of each sample was measured by the BCA Protein sssay kit (Thermo Fisher Scientific, Inc.). Then $10 \mu \mathrm{g}$ of protein lysed buffer were subjected to a $10 \%$ SDS-PAGE and transferred to a PVDF membrane (Millipore, MA, USA). Following blocking with $5 \%$ non-fat milk for $2 \mathrm{~h}$ at room temperature, membranes were incubated with specific primary antibody at $4^{\circ} \mathrm{C}$ overnight (KMT5A: 1:2,000, Abcam, \#ab3798, Cambridge, MA, USA; CDH1: 1:2,000, \#14472, Cell Signaling Technology, Inc., Danvers, MA, USA; $\beta$-actin: 1:10,000, Thermo Fisher Scientific, Inc., \#MA5-15739-HRP). Following washing with TBST three times, membranes were incubated with HRP-conjugated secondary antibody (1:5,000, Cell Signaling Technology, \#7076, Inc., Danvers, MA, USA) for $1 \mathrm{~h}$ at room temperature. Then proteins were photographed using ECL detection reagents (Thermo Fisher Scientific, Inc.) and detected with a Bio-Rad GelDoc XR+ system (Bio-Rad Laboratories, Inc., Hercules, CA, USA).

Luciferase reporter assay. Dual Luciferase Report Assay were performed according to the manufacturer's protocol (Dual-Glo ${ }^{\circledR}$ Luciferase assay system, \#E2940, Promega Corporation, Madison, WI, USA). In brief, $0.6 \mu \mathrm{g}$ luciferase reporter and were cotransfected along with $0.3 \mu \mathrm{g} \mathrm{pCMV-lacZ}$ (Promega Corporation) plasmid into the 786-O cells (SCR and shKMT5A). At $48 \mathrm{~h}$ following transfection, these cells were harvested and lysed in 1x reporter lysis buffer (Promega Corporation). Luciferase activities were determined with luciferase assay system (Promega Corporation) according to the manufacturer's protocol. The method of normalization was the Firefly comparison with Renilla luciferase activity. 

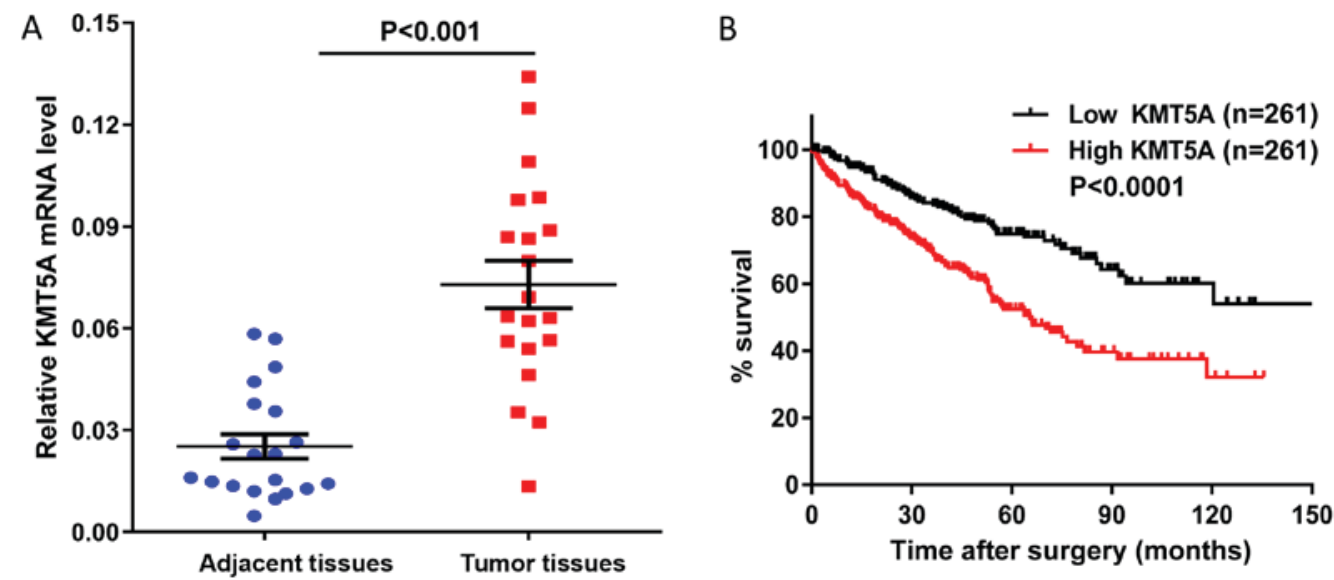

Figure 1. High expression of KMT5A is associated with shorter OS time in ccRCC. (A) Relative KMT5A mRNA level was significantly upregulated in ccRCC tissues compared with adjacent normal tissues, using a reverse transcription-quantitative polymerase chain reaction assay. (B) Kaplan-Meier curves of OS time in high and low KMT5A expression ccRCC groups. The OS time was significantly decreased in the high KMT5A expression group compared with the low expression group (log-rank test; P<0.0001). The ccRCC data were obtained from The Cancer Genome Atlas database (https://tcga-data.nci.nih.gov/publications/tcga). KMT5A, N-lysine methyltransferase KMT5A; ccRCC, clear cell renal cell carcinoma; OS, overall survival.

Chromatin immunoprecipitation (CHIP) assay. Chromatin immunoprecipitation was performed following a published protocol (24). Antibody against KMT5A (Abcam) was used to immunoprecipitate sonicated chromatins prepared from 786-O cells. Five percent of each post-sonication sample was saved as the input control and pre-immune IgG was used for specificity control. DNA extracted from precipitated chromatins were quantitated using qrtPCR in triplicates with primers for CDH1 promoter (forward, 5'-GCAGGTCCCATA ACCCACCT-3'; and reverse, 5'-CATAGACGCGGTGACCCT CT-3'). DNA extracted from saved input were quantitated in parallel (Ct[Input]), and results from IP by anti-KMT5A mAb or pre-immnue $\mathrm{IgG}(\mathrm{Ct}[\mathrm{IP}])$ were then used to calculate relative specific occupancy or non-specific background using the equation: $2^{\wedge}(\mathrm{Ct}[\mathrm{IP}]-\mathrm{Ct}[$ Input $]) \times 100 \%$.

Statistical analysis. Statistical analysis was performed with SPSS version 22.0 (IBM Corp., Armonk, NY, USA). The Kaplan-Meier analysis with log-rank test, Pearson's test, Student's t-test One-way ANOVA and Bonferroni's post-hoc test were used for compare the differences between the variables. $\mathrm{P}<0.05$ was considered to indicate statistically significant differences.

\section{Results}

High KMT5A expression is associated with shorter OS time in $c c R C C$. Firstly, the KMT5A mRNA levels were detected in $20 \mathrm{ccRCC}$ and paired adjacent renal tissue specimens from the First Hospital of Quanzhou Affiliated to Fujian Medical University. The KMT5A mRNA levels were significantly upregulated in the ccRCC tissues compared with those in the adjacent normal tissues (Fig. 1A). Subsequently, the KMT5A expression levels in the postoperative tumor tissues obtained from 522 patients with ccRCC were analyzed. These samples were grouped according to the KMT5A mRNA level (high KMT5A group: The expression range from 500.9 to 1870.24 , $\mathrm{n}=261$ and low KMT5A group: The expression range from 150.65 to $500.8, n=261$ ) and the association between that and the OS time of the patients was assessed using Kaplan-Meier analysis. The OS time was significantly poorer in the high-expression group compared with the low-expression group (Fig. 1B). Together, these findings suggest that KMT5A serves a role in ccRCC development.

KMT5A knockdown hinders RCC cell migration and invasiveness. In order to evaluate the function of KMT5A in RCC cell migration and invasiveness in vitro, ccRCC and papillary RCC cells with silenced KMT5A expression (786-O-shKMT5A and ACHN-shKMT5A) and their corresponding controls (786-O-SCR and ACHN-SCR) were used. Transwell assays were performed to assess the migration and invasion ability of the cells. The cells with silenced KMT5A expression displayed sharp declines in cell migration and invasiveness compared with the corresponding control groups, which was prevented by re-expression of KMT5A (Fig. 2A-D). These data demonstrate that KMT5A enhances RCC cell migration and invasiveness in vitro.

KMT5A downregulates CDH1 expression. In order to explore whether KMT5A activates EMT in order to promote ccRCC metastasis, the effect of KMT5A on CDH1, a central participating factor in EMT, was investigated. Firstly, the question of whether KMT5A decreases CDH1 protein levels in ccRCC cells was addressed. Western blot analysis was used to determine any effects on the $\mathrm{CDH} 1$ protein expression levels in the KMT5A-knockout and control cells, and revealed that knockdown of KMT5A expression markedly upregulated CDH1 protein levels in 786-O cells (Fig. 3A). Next, the CDH1 mRNA levels were measured, demonstrating that the knockdown of KMT5A increased CDH1 transcription levels $(\mathrm{P}<0.01$; Fig. 3B). Furthermore, the luciferase reporter assay revealed that silencing KMT5A in 786-O cells led to the activation of $\mathrm{CDH} 1$ promoter activity $(\mathrm{P}<0.01$; Fig. $3 \mathrm{C})$. Finally, the chromatin immunoprecipitation (ChIP) assay demonstrated that immunoprecipitation using an anti-KMT5A antibody on chromatin fragments from 786-O cells specifically enriched CDH1 promoter sequences $(\mathrm{P}<0.01$; Fig. $3 \mathrm{D})$, indicating that 
A

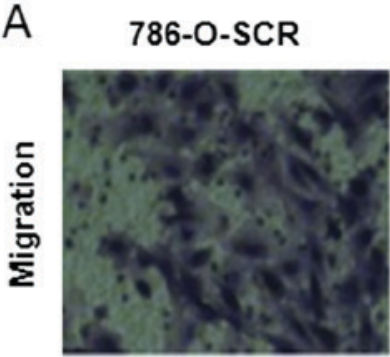

\section{6-O-shKMT5A}

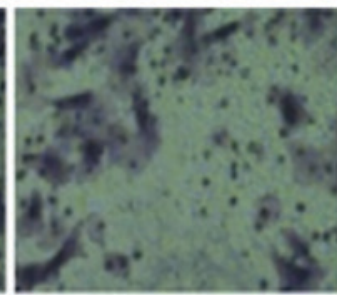

B
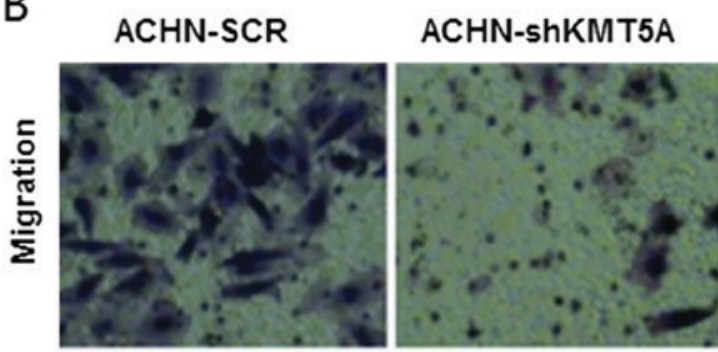

C

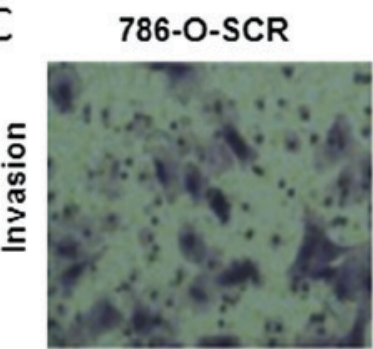

D

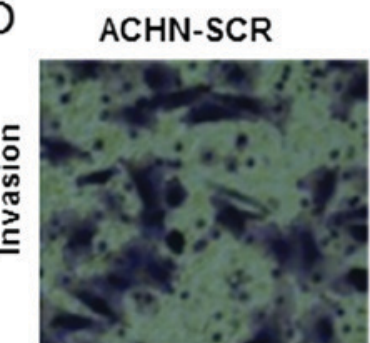

786-O-shKMT5A

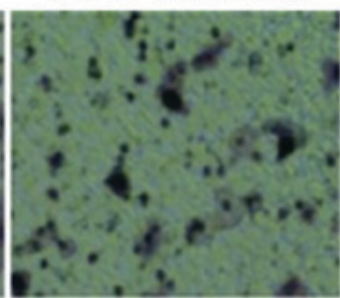

ACHN-shKMT5A

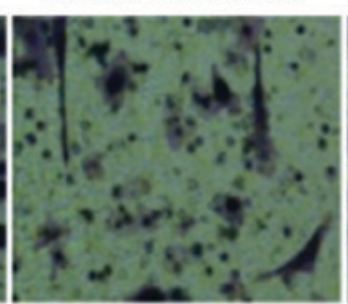

786-O-shKMT5A-RES

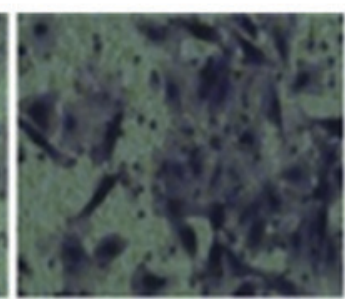

ACHN-ShKMT5A-RES

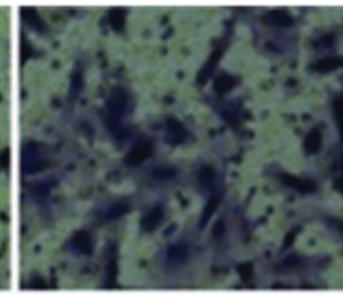

786-O-shKMT5A-RES

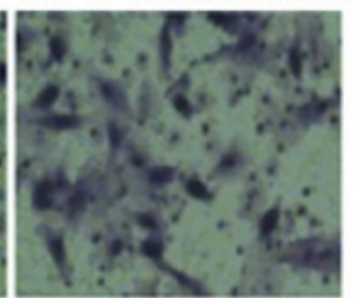

ACHN-shKMT5A-RES

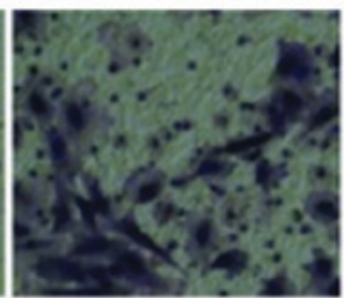

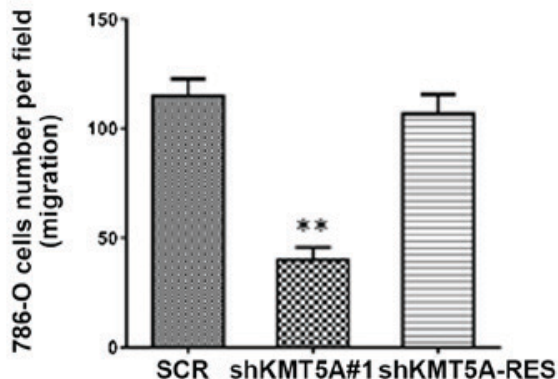
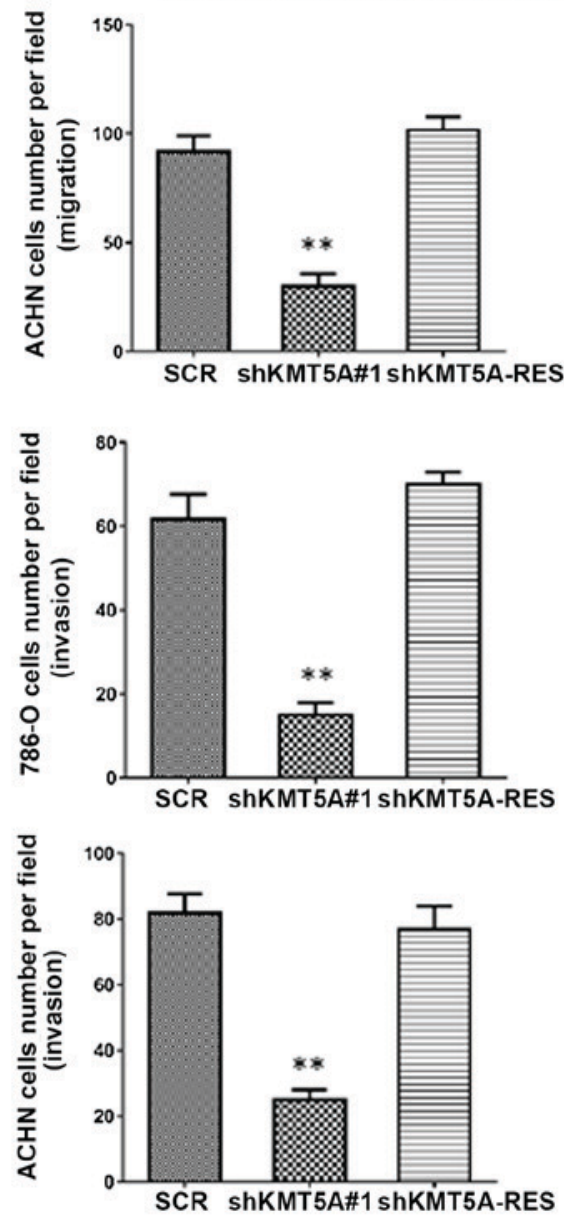

Figure 2. KMT5A improves ccRCC cell migration and invasion. Representative Transwell migration assay images and quantification of migratory cells for renal call carcinoma (A) 786-O and (B) ACHN cells, which had been transfected with SCR sequence, had the KMT5A expression silenced with shKMT5A, and had the KMT5A expression rescued with shKMT5A-RES. Representative Transwell invasion assay images and quantification of invasive cells for renal call carcinoma (C) 786-O and (D) ACHN cells, which had been transfected with SCR sequence, had the KMT5A expression silenced with shKMT5A, and had the KMT5A expression rescued with shKMT5A-RES. Significant differences were confirmed by one-way analysis of variance. ${ }^{* * *} \mathrm{P}<0.01$, shKMT5A group compared with the SCR group. KMT5A, N-lysine methyltransferase KMT5A; SCR, scrambled control; shKMT5A, short hairpin RNA targeting KMT5A, shKMT5A-RES.

endogenous KMT5A is associated with the $\mathrm{CDH} 1$ promoter in the ccRCC cells. These data collectively indicate that KMT5A downregulates $\mathrm{CDH} 1$ transcription in the ccRCC cells, likely via the $\mathrm{CDH} 1$ promoter.

Prognostic value of KMT5A and CDH1 for patients with $c c R C C$. Following the finding of upregulation in the expression level of CDH1 in the 786-O cells upon KMT5A knockdown, the correlation between the expression levels of KMT5A and $\mathrm{CDH} 1$ in the tumor tissues from the aforementioned patients with ccRCC was investigated. High levels of CDH1 (high CDH1 group: The expression range from 1949.34 to 39322.42 , n=261 and low CDH1 group: The expression range from 3.37 to $1939.41, \mathrm{n}=261)$ were observed in $30.3 \%(79 / 261$ samples) of the high-KMT5A group in comparison with $69.0 \%(180 / 261$ samples) in the low-KMT5A group ( $<<0.001$; Fig. 4A). Furthermore, a negative correlation was revealed between the expression levels of KMT5A and CDH1 in the ccRCC samples $(\mathrm{r}=-0.6506 ; \mathrm{P}<0.001$; Fig. 4B). The patients whose samples expressed high CDH1 levels had longer OS times compared with those whose samples expressed low levels (Fig. 4C). The patients with high KMT5A and low CDH1 expression levels were associated with the shortest OS times, and those with low KMT5A and high CDH1 expression levels were associated with the longest (Fig. 4D). The combination of KMT5A and $\mathrm{CDH} 1$ was demonstrated to be a promising independent 
A

KMT5A
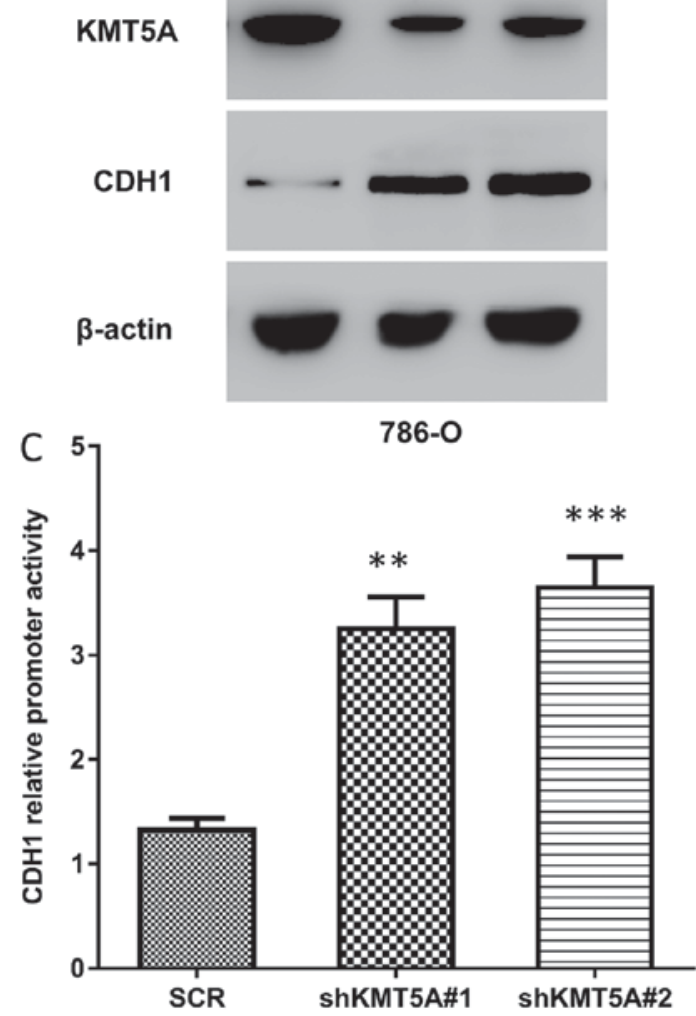

B

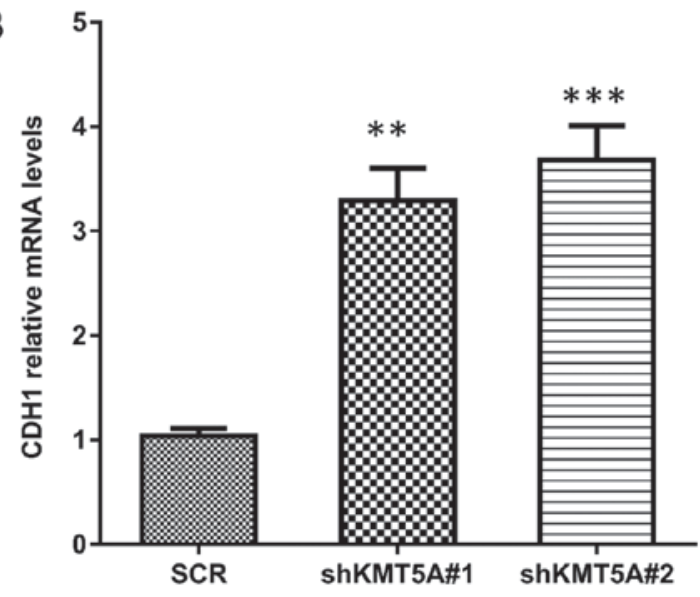

D

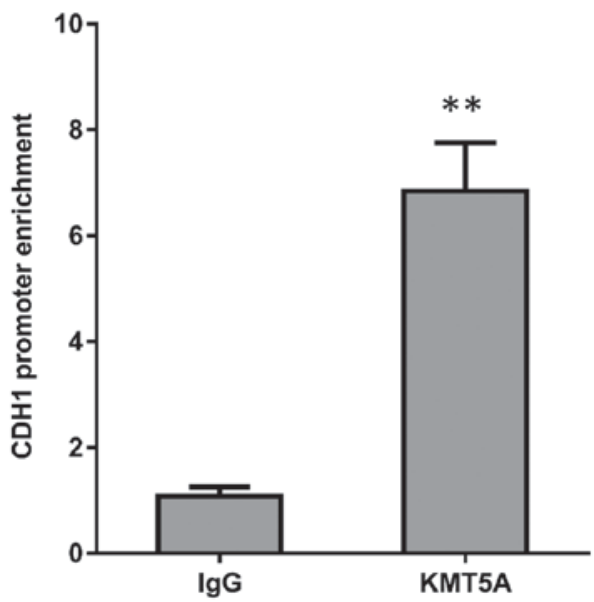

Figure 3. KMT5A downregulates CDH1 expression by inhibiting its transcription. (A) Western blot images demonstrating that knockdown of KMT5A expression level in 786-O cells led to the upregulation CDH1 protein levels. (B) Knockdown of KMT5A expression in 786-O cells increased the relative CDH1 mRNA levels, detected using a reverse transcription-qPCR assay. The means \pm SD of relative mRNA levels from three independent experiments are represented. (C) KMT5A knockdown led to an increase in the CDH1 promoter activity in 786-O cells. The means \pm SD of normalized luciferase activity from three independent experiments are represented. (D) Endogenous KMT5A was associated with the CDH1 promoter. Chromatin immunoprecipitation-qPCR was performed on sonicated chromatin from 786-O cells immunoprecipitated with anti-KMT5A antibody or isotype IgG. The CDH1 promoter segments were measured by qPCR against a $5 \%$ input. The means \pm SD from three independent experiments are represented. Significant differences were confirmed by Student's t-test for comparing 2 groups or one-way analysis of variance for $>2$ groups. ${ }^{* * *} \mathrm{P}<0.01$ and ${ }^{* * * *} \mathrm{P}<0.001$, each group compared vs. the control group. KMT5A, N-lysine methyltransferase KMT5A; SCR, scrambled control; shKMT5A, short hairpin RNA targeting KMT5A; CDH1, cadherin-1; qPCR, quantitative polymerase chain reaction; $\mathrm{SD}$, standard deviation.

prognostic indicator for OS time $(\mathrm{P}<0.001)$, more accurate than KMT5A or CDH1 alone.

\section{Discussion}

ccRCC is one of the most common types of kidney cancer worldwide, and is associated with poor prognosis owing to high rates of metastasis and recurrence (25-27). The underlying molecular mechanism in ccRCC metastasis is not well known. Numerous studies have reported that the EMT is an important process in ccRCC cell metastasis (28-31). In the present study, supporting evidence was collected for the hypothesis that KMT5A enhances EMT through inhibiting CDH1 transcription levels, and consequently inducing ccRCC cells metastasis. Consistent with these findings, ccRCC patients with high KMT5A expression levels were associated with shorter OS time. Overall, KMT5A is identified as an important factor in ccRCC cell metastasis.

Previous studies demonstrated that KMT5A serves a vital role in tumor development and progression (12-22). To the best of our knowledge, the association between KMT5A and ccRCC has not been reported to date. In the present study, it was confirmed that the KMT5A expression level was significantly upregulated in ccRCC tissues compared with the adjacent normal tissues. Furthermore, ccRCC patients with KMT5A-high expression levels in their tumor tissue were revealed to be associated with shorter postoperative OS time. Together, these data suggest that KMT5A may be a novel target for ccRCC treatment.

The epithelial-mesenchymal transition (EMT) is considered to be the initial step in ccRCC metastasis (28) and CDH1 is a central factor in this mechanism (29-31). At present, no studies have reported on the association between KMT5A and CDH1 in ccRCC, to the best of our knowledge. The results of the present study demonstrated that the knockdown of KMT5A expression inhibits the migration and invasiveness of ccRCC 786-O cells. In order to investigate the molecular mechanism underlying the promotional effects of KMT5A on ccRCC metastasis, the present investigated the relationship between KMT5A and CDH1. The knockdown of KMT5A expression 
A

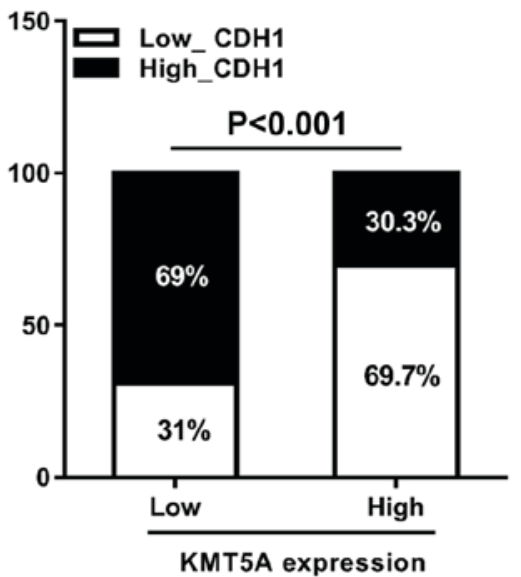

C

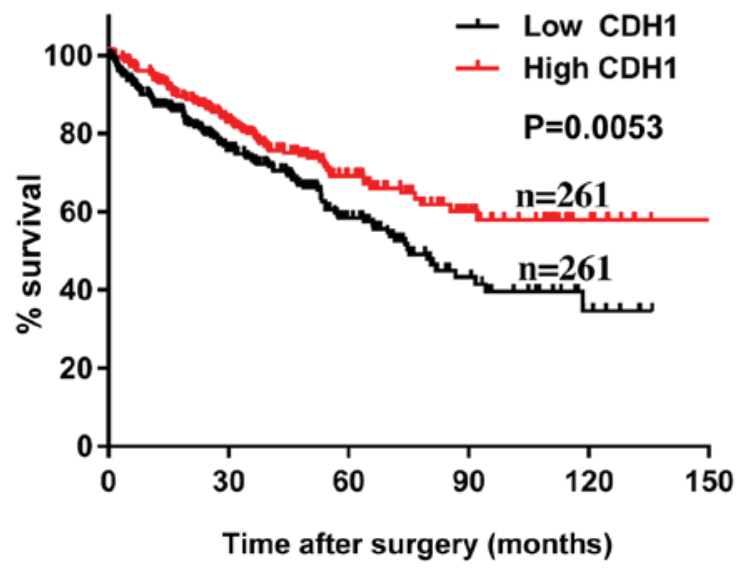

B

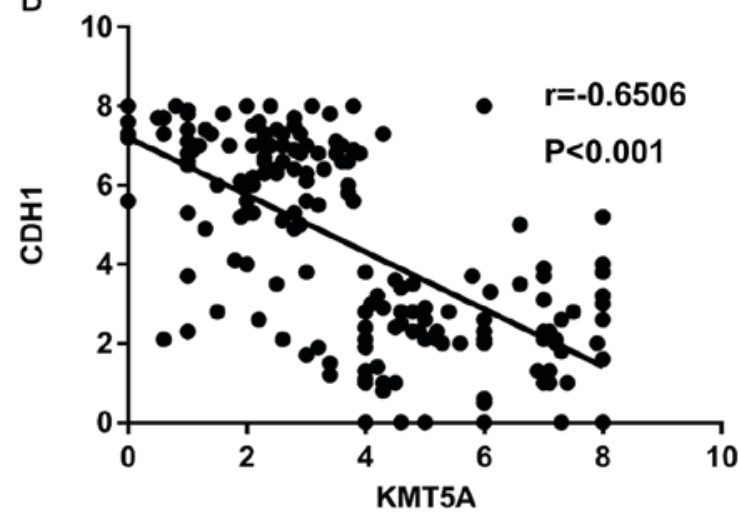

D

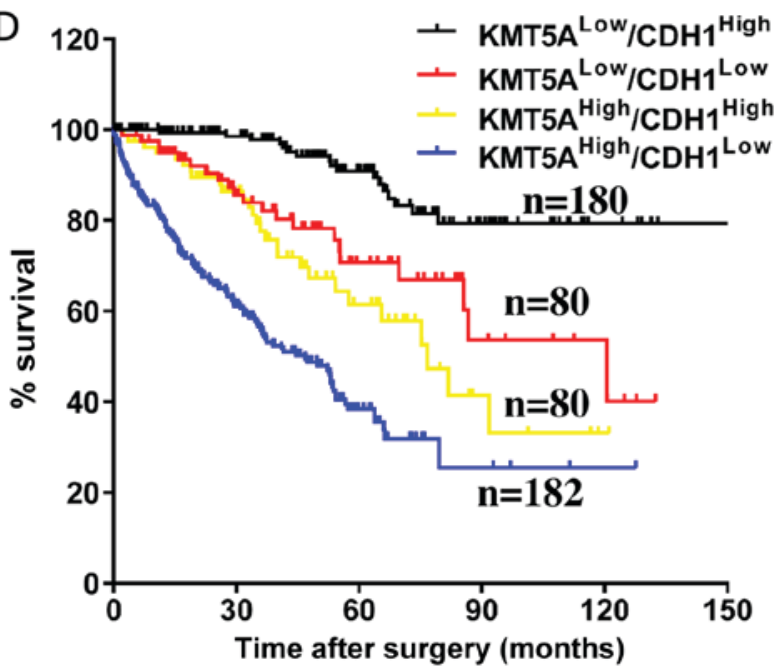

Figure 4. Prognostic values of KMT5A and CDH1 for patients with ccRCC, assessed by Kaplan-Meier analyses. (A) High levels of CDH1 were observed in $30.3 \%$ of the high-KMT5A group in comparison with $69.0 \%$ in the low-KMT5A group. (B) A negative correlation was revealed between the expression levels of KMT5A and CDH1 in ccRCC tissues ( $\mathrm{r}=-0.6506$; $\mathrm{P}<0.001)$. (C) The patients with ccRCC who exhibited high CDH1 expression were associated with longer OS time compared with the low CDH1 expression group. (D) Patients who had high KMT5A as well as low CDH1 expression levels were associated with the poorest prognosis (the shortest OS time), whereas those with low KMT5A and high CDH1 expression levels had the best prognosis. The ccRCC data was obtained from The Cancer Genome Atlas database (https://tcga-data.nci.nih.gov/publications/tcga). ccRCC, clear cell renal cell carcinoma; KMT5A, N-lysine methyltransferase KMT5A; CDH1, cadherin-1; OS, overall survival.

in the 786-O cells upregulated $\mathrm{CDH} 1 \mathrm{mRNA}$ and protein levels. Furthermore, downregulation of KMT5A increased $\mathrm{CDH} 1$ promoter luciferase activity in 786-O cells. Finally, the ChIP assay revealed that endogenous KMT5A was associated with the CDH1 promoter in the ccRCC cells. Together, these findings indicate that KMT5A may be an important factor in ccRCC cell migration and invasion by inhibiting CDH1 transcription.

ccRCC is associated with a markedly poor prognosis due to high rates of metastasis and recurrence. At present, no biomarkers are available for ccRCC diagnosis and prediction. The present findings demonstrated that KMT5A expression level was significantly upregulated in ccRCC tissues compared with adjacent normal tissues. Patients with high KMT5A expression levels were revealed to be associated with shorter postoperative OS time. In agreement with the clinical results, in vitro experiments demonstrated that KMT5A enhances ccRCC cell migration and invasion by inhibiting CDH1 expression. Patients with ccRCC who exhibited high KMT5A and low CDH1 levels had the poorest prognosis, with the shortest OS time, and those with low KMT5A and high CDH1 expression had the best prognosis. The combination of KMT5A and CDH1 was confirmed to be an independent prognostic indicator for OS $(\mathrm{P}<0.001)$, which was more accurate than monitoring KMT5A or CDH1 expression alone. Consequently, KMT5A serves an important role in ccRCC development and progression, and it may be a novel target for ccRCC treatment and prevention.

\section{Acknowledgements}

Not applicable.

Funding

No funding was received.

\section{Availability of data and materials}

The datasets used and/or analyzed during the present study are available from the corresponding author on reasonable request. 


\section{Authors' contributions}

ZZL, DSM, YBC, JMZ, HHC and JJJ performed the experiments and analyzed the data. ZZL and ZSZ designed the experiments and wrote the manuscript.

\section{Ethics approval and consent to participate}

The human sample collection was approved by the ethical committee of the First Hospital of Quanzhou Affiliated to Fujian Medical University. Patients provided written informed consent.

\section{Patient consent for publication}

Not applicable.

\section{Competing interests}

The authors declare that they have no competing interests.

\section{References}

1. Chow WH, Dong LM and Devesa SS: Epidemiology and risk factors for kidney cancer. Nat Rev Urol 7: 245-257, 2010.

2. Siegel RL, Miller KD and Jemal A: Cancer statistics. CA Cancer J Clin 68: 7-30, 2018.

3. Martinez-Salamanca JI, Huang WC, Millan I, Bertini R, Bianco FJ, Ciancio G, Hernández C, Herranz F, Haferkamp A, et al: Prognostic impact of the 2009 UICC/AJCC TNM staging system for renal cell carcinoma with venous extension. Eur Urol 59: 120-127, 2011.

4. Fang J, Feng Q, Ketel CS, Wang H, Cao R, Xia L, Erdjument-Bromage H, Tempst P, Simon JA and Zhang Y: Purification and functional characterization of SET8, a nucleosomal histone H4-lysine 20-specific methyltransferase. Curr Biol 12: 1086-1099, 2002

5. Nishioka K, Rice JC, Sarma K, Erdjument-Bromage H, Werner J, Wang Y, Chuikov S, Valenzuela P, Tempst P, Steward R, et al: PR-Set7 is a nucleosome-specific methyltransferase that modifies lysine 20 of histone $\mathrm{H} 4$ and is associated with silent chromatin. Mol Cell 9: 1201-1213, 2002.

6. Oda H, Okamoto I, Murphy N, Chu J, Price SM, Shen MM, Torres Padilla ME, Heard E and Reinberg D: Monomethylation of histone H4-lysine 20 is involved in chromosome structure and stability and is essential for mouse development. Mol Cell Biol 29: 2278-2295, 2009.

7. Houston SI, McManus KJ, Adams MM, Sims JK, Carpenter PB, Hendzel MJ and Rice JC: Catalytic function of the PR-Set7 histone H4 lysine 20 monomethyltransferase is essential for mitotic entry and genomic stability. J Biol Chem 283: 19478-19488, 2008.

8. Jorgensen S, Elvers I, Trelle MB, Menzel T, Eskildsen M, Jensen ON, Helleday T, Helin K and Sorensen CS: The histone methyltransferase SET8 is required for S-phase progression. J Cell Biol 179: 1337-1345, 2007.

9. Abbas T, Shibata E, Park J, Jha S, Karnani N and Dutta A CRL4(Cdt2) regulates cell proliferation and histone gene expression by targeting PR-Set7/Set8 for degradation. Mol Cell 40 9-21, 2010.

10. Centore RC, Havens CG, Manning AL, Li JM, Flynn RL, Tse A, Jin J, Dyson NJ, Walter JC and Zou L: CRL4(Cdt2)-mediated destruction of the histone methyltransferase Set8 prevents premature chromatin compaction in S phase. Mol Cell 40: 22-33, 2010.

11. Wu S, Wang W, Kong X, Congdon LM, Yokomori $\mathrm{K}$, Kirschner MW and Rice JC: Dynamic regulation of the PR-Set7 histone methyltransferase is required for normal cell cycle progression. Genes Dev 24: 2531-2542, 2010.

12. Guo Z, Wu C, Wang X, Wang C, Zhang R and Shan B: A polymorphism at the miR-502 binding site in the 3'-untranslated region of the histone methyltransferase SET8 is associated with hepatocellular carcinoma outcome. Int J Cancer 131: 1318-1322, 2012.
13. Yang F, Sun L, Li Q, Han X, Lei L, Zhang H and Shang Y: SET8 promotes epithelial-mesenchymal transition and confers TWIST dual transcriptional activities. EMBO J 31: 110-123, 2012.

14. Song F, Zheng H, Liu B, Wei S, Dai H, Zhang L, Calin GA, Hao X, Wei Q, Zhang W and Chen K: An miR-502-binding site single-nucleotide polymorphism in the 3'-untranslated region of the SET8 gene is associated with early age of breast cancer onset. Clin Cancer Res 15: 6292-6300, 2009.

15. Yu N, Huangyang P, Yang X, Han X, Yan R, Jia H, Shang Y and Sun L: microRNA-7 suppresses the invasive potential of breast cancer cells and sensitizes cells to DNA damages by targeting histone methyltransferase SET8. J Biol Chem 288: 19633-19642, 2013.

16. Liu B, Zhang X, Song F, Zheng H, Zhao Y, Li H, Zhang L, Yang M, Zhang W and Chen K: MiR-502/SET8 regulatory circuit in pathobiology of breast cancer. Cancer Lett 376: 259-267, 2016.

17. Mosallayi M, Simonian M, Khosravi S, Salehi AR, Khodadoostan M, Sebghatollahi V, Baradaran A and Salehi R: Polymorphism (rs16917496) at the miR-502 binding site of the lysine methyltransferase 5A (SET8) and Its correlation with colorectal cancer in iranians. Adv Biomed Res 6: 77, 2017.

18. Liao T, Wang YJ, Hu JQ, Wang Y, Han LT, Ma B, Shi RL, Qu N, Wei WJ, Guan Q, et al: Histone methyltransferase KMT5A gene modulates oncogenesis and lipid metabolism of papillary thyroid cancer in vitro. Oncol Rep 39: 2185-2192, 2018.

19. Wang C, Wu J, Zhao Y and Guo Z: miR-502 medaited histone methyltransferase SET8 expression is associated with outcome of esophageal squamous cell carcinoma. Sci Rep 6: 32921, 2016.

20. Benamar M, Guessous F, Du K, Corbett P, Obeid J, Gioeli D, Slingluff CL Jr and Abbas T: Inactivation of the CRL4-CDT2-SET8/p21 ubiquitylation and degradation axis underlies the therapeutic efficacy of pevonedistat in melanoma. EBioMedicine 10: 85-100, 2016.

21. Yao L, Li Y, Du F, Han X, Li X, Niu Y, Ren S and Sun Y: Histone H4 Lys 20 methyltransferase SET8 promotes androgen receptor-mediated transcription activation in prostate cancer. Biochem Biophys Res Commun 450: 692-696, 2014.

22. Wang C, Guo Z, Wu C, Li Y and Kang S: A polymorphism at the miR-502 binding site in the 3' untranslated region of the SET8 gene is associated with the risk of epithelial ovarian cancer. Cancer Genet 205: 373-376, 2012.

23. Livak KJ and Schmittgen TD: Analysis of relative gene expression data using realtime quantitative PCR and the 2(Delta Delta C(T)) method. Methods 25: 402-408, 2001.

24. Lee TI, Johnstone SE and Young RA: Chromatin immunoprecipitation and microarray-based analysis of protein location. Nat Protoc 1: 729-748, 2006.

25. Siegel R, Naishadham D and Jemal A: Cancer statistics, 2012. CA Cancer J Clin 62: 10-29, 2012.

26. Janzen NK, Kim HL, Figlin RA and Belldegrun AS: Surveillance after radical or partial nephrectomy for localized renal cell carcinoma and management of recurrent disease. Urol Clin North Am 30: 843-852, 2003.

27. Jemal A, Siegel R, Ward E, Murray T, Xu J, Smigal C and Thun MJ: Cancer statistics, 2006. CA Cancer J Clin 56: 106-130, 2006

28. Myszczyszyn A, Czarnecka AM, Matak D, Szymanski L, Lian F, Kornakiewicz A, Bartnik E, Kukwa W, Kieda C and Szczylik C: The role of hypoxia and cancer stem cells in renal cell carcinoma pathogenesis. Stem Cell Rev 11: 919-943, 2015.

29. Krishnamachary B, Zagzag D, Nagasawa H, Rainey K, Okuyama H, Baek JH and Semenza GL: Hypoxia-inducible factor-1-dependent repression of E-cadherin in von HippelLindau tumor suppressor-null renal cell carcinoma mediated by TCF3, ZFHX1A, and ZFHX1B. Cancer Res 66: 2725-2731, 2006.

30. Russell RC and Ohh M: The role of VHL in the regulation of E-cadherin: A new connection in an old pathway. Cell Cycle 6: 56-59, 2007.

31. Harten SK, Shukla D, Barod R, Hergovich A, Balda MS, Matter K, Esteban MA and Maxwell PH: Regulation of renal epithelial tight junctions by the von Hippel-Lindau tumor suppressor gene involves occludin and claudin 1 and is independent of E-cadherin. Mol Biol Cell 20: 1089-1101, 2009. International (CC BY-NC-ND 4.0) License. 